\title{
Clinical Laboratory Educators' Conference 2010 Abstracts
}

\author{
BILOXI, MS
}

\begin{abstract}
The following abstracts were presented during the 2010 American Society for Clinical Laboratory Science (ASCLS) Clinical Laboratory Educators' Conference February 25-27, 2010 in Biloxi, Mississippi. Abstracts are reviewed by appropriate representatives of the ASCLS Educational Scientific Assembly. They are the final authority in selecting or rejecting an abstract.
\end{abstract}

\section{POSTER PRESENTATIONS}

Analysis of Resistance Education for Technologists using Antibiotic Sensitivity Testing

Roxanne Alter, MS, University of Nebraska Medical Center, Omaha, NE

A workshop was designed to help prepare practicing Clinical Laboratory Scientists with the detection of emerging pathogens in the Microbiology section of the clinical laboratory. This educational tool was intended to address the lack of basic knowledge of resistance mechanisms of common pathogens identified routinely in the microbiology laboratory. A previous survey identified half of the bench technologists working in microbiology in Nebraska could not identify basic resistance patterns and or the need for additional testing. This workshop was designed with practical hands on experience in identifying the detection of $\beta$-lactam resistance in Gram negative rods. A pre-session test of 10 questions was put on an educational program called Blackboard and participants took this test prior to attendance of the workshop. Questions were designed to identify the knowledge level of the audience. Participants had a minimum of five years with several more than thirty years experience in a microbiology laboratory. These multiple choice questions were scored automatically and corrective feedback was given. Twenty-one individuals took the pre-test and more than half of these individuals scored fifty percent or less. Questions as basic as how does the antibiotic penicillin work were consistently missed. The educational intervention included three hours of lecture and three hours of laboratory evaluation. A post-test was administered one week following the workshop with the same format as the pre-test. This time 11 case-based questions were asked and the average score was 82 percent. An improvement of basic knowledge was evident with this post test evaluation.

Applying Evidence-Based Methods to Laboratory Medicine Decision-Making Diana Mass, MA, MT(ASCP), Arizona State University, Tempe, AZ

72 VOL 23, NO 2 SPRING 2010 CLINICAL LABORATORY SCIENCE
Initiated in 2006, the Laboratory Medicine Best Practices (LMBP) project is a CDC (Centers for Disease Control and Prevention) sponsored initiative. The goal is to develop and pilot evidence-based methods to conduct systematic reviews of the effectiveness of laboratory medicine practices. Evidence-based methods have become the standard practice in healthcare. Most evidence reviews rely on published peer-reviewed literature; however, published evidence of practice effectiveness is limited in laboratory medicine. Therefore, these new LMBP systematic review methods also include the evaluation of unpublished quality improvement evidence projects conducted in many laboratory settings using the same criteria applied to the published literature. Results of these reviews will be used to issue evidence-based recommendations which can inform laboratory professionals on what practices are effective and in what settings. An understanding of evidence-based approaches in decision making can contribute to quality improvement study design and documentation. Through the exchange of information, a laboratory's quality improvement efforts can also contribute to improving patient care and outcomes. Educators need to include this evidence-based methodology in their quality improvement/ management curriculum. In addition, research/ quality improvement activities need to follow this methodology to provide effective decisions in laboratory practice. The LMBP initiative has been endorsed by major laboratory accrediting and professional organizations including The Joint Commission, College of American Pathologists (CAP), Consortium Office Laboratory Accreditation (COLA), American Society for Clinical Laboratory Sciences (ASCLS), Clinical Laboratory Management Association (CLMA), American Society of Clinical Pathology (ASCP), and National Association of Clinical Biochemistry (NACB).

The Benefits of Clinical Laboratory Science Faculty Teaching an Undergraduate Public Health Course

Linda J. Laatsch, PhD, MT(ASCP)SM, Marquette University, Milwaukee, WI

Public Health courses are typically taught in graduate schools or medical schools. However, undergraduate students who might be interested in public health as a career may not even be aware of this field. In 2002, Marquette University's Clinical Laboratory Science Department offered a 3-credit Public Health course to undergraduates for the first time. This course has been offered each spring semester since then, and to date 283 students have been enrolled. The course is open to all students, but the majority are College of Health Sciences majors. Many benefits to both students and the Clinical Laboratory Science Department have been realized over the years. The course has contributed to student interest in and 


\section{CLINICAL PRACTICE}

knowledge of public health as a career option. Since the instructor is a clinical laboratory scientist she is able to emphasize the importance of laboratory medicine in both public health and healthcare in general. This is useful information for health science students, many of whom are pre-med or pre-physician assistant majors. Additionally, the course has prompted some students to transfer into the Clinical Laboratory Science major or to enroll in other courses offered by our Department. Other benefits, including fiscal advantages of offering this course through the Clinical Laboratory Science Department, will be discussed. Development and implementation of the course will also be presented as a model that could be followed by other Clinical Laboratory Science educators.

Development of an Online Transitional Degree Completion Program in Medical Laboratory Science to Address the Growing Shortage of Medical Laboratory Scientists in Oklahoma

Marvita McGuire, PhD, MT (ASCP), Northeastern State University, Tahlequah, $\mathrm{OK}$

The growing need for medical laboratory scientists in Oklahoma is due to three predominant factors: an aging population, retirement of the existing workforce of medical laboratory scientists and a shortage of schools of medical technology. In response to this problem, Northeastern State University is developing an online transitional degree completion program. The program emphasizes the education of currently employed, certified medical laboratory technicians as medical laboratory scientists. Students complete upper division coursework online while fulfilling the clinical requirements in their current places of employment. Completion of the program satisfies the requirements for a bachelor's degree in medical laboratory science as well as eligibility requirements for the ASCP Board of Certification exam. The Transitional degree completion program was approved by the Oklahoma State Regents of Higher Education on June 30, 2009 and sixteen MLTs were accepted for the program in August, 2009. In accordance with the National Accreditation Agency for Clinical Laboratory Science (NAACLS), NSU established clinical site affiliation agreements with the student's employer as well as providing Clinical Guide manuals. Each student/employee was given a Student Guide manual containing checklists, policies, textbook lists and evaluation of cognitive, attitudinal and psychomotor skills. Preparation of preliminary report documents for submission to NAACLS are in process. The program is designed to be completed in six semesters and the first graduating class is anticipated for the summer of 2011. The poster will include a summary of the transitional program development as well as a comparison of consortium based programs in Oklahoma.

\section{The Efficacy of Essential Functions on Clinical Laboratory Science Programs}

Maria E. Delost, Ph.D., CLS(NCA), Youngstown State University, Youngstown, OH; Teresa Nadder, Ph.D., CLS (NCA), Virginia Commonwealth University, Richmond, VA

Essential functions are nonacademic program requirements originally mandated by the National Accrediting Agency for Clinical Laboratory Sciences (NAACLS) in the 1986 Essentials. These functions include task-oriented physical and behavioral standards in which students must engage to successfully complete a laboratory program. Essential functions describe requirements related to vision, communication skills, physical and motor skills, psychological stability, behavioral, intellectual and integrative skills. Additionally, programs may require a physical examination to assess a student's ability to meet essential requirements. Essential functions may be subjective in nature and may be difficult to implement while considering compliance with the American with Disabilities Act (ADA). This study investigated the efficacy of essential functions in NAACLS accredited and approved programs. An electronic survey was sent to 564 programs with 267 responses (47.3\% response rate). Essential functions are distributed to applicants in 255 (95.5\%) of the programs and concerns are discussed with the students (222, $83.1 \%)$. However, only $103(38.6 \%)$ of the participants responded that essential functions are required by both the $\mathrm{ADA}$ and NAACLS. Physical examinations are required by $146(54.7 \%)$ of the programs, yet reviewed with students by only 109 (40.8\%) of the respondents. Of the respondents, $213(80.0 \%)$ stated that a student had never been removed from a program based on essential functions. Students with a variety of documented disabilities have successfully completed laboratory programs. Participants felt most comfortable referring students for academic coaching (257, 96.2\%) when compared to medical concerns $(231,86.5 \%)$, and psychological concerns $(221,82.7 \%)$.

\section{Electronic Self Study}

Martha J. Lake, EdD, MT(ASCP), West Virginia University, Morgantown, WV; James L. Vossler, MS, MT(ASCP)SM, SUNY Upstate Medical University, Syracuse, NY

West Virginia University (WVU) CLS Program prepared and submitted the first electronic self study to NAACLS. In response to inquiries about the possibility of submitting the self study in electronic form, NAACLS' Review Committee for Accredited Programs developed a format for submitting an electronic self study. The CLS program at WVU volunteered to submit their Self Study document in this new format. The format was first designed as a series of Microsoft Word forms and later adapted to Adobe forms. Each standard is addressed in a separate form. The forms allow the attachment of Word, Adobe or Excel documents and links to web pages. As most of our documents already exist in an electronic form, it was a simple task to attach them to the appropriate form. In cases where the documents were not electronic, they were scanned into Adobe documents which were then attached to the appropriate form. The preparation of the electronic self study was surprisingly easy and faster than preparing a paper copy. It was also much easier to share the document with faculty and administrators by posting it on a shared drive. The electronic self study was reviewed and both NAACLS staff and the volunteer paper reviewer found the format easy to navigate and use. NAACLS is seeking to implement the electronic self study by allowing programs the option to submit electronic or paper copies with the hope that eventually all self study documents will be submitted in the electronic format.

How to Maintain University-Based CLS Program Viability! Julie Hammerling, MS, CLS(NCA), MT(ASCP), Brenda Van Der Heyden, CLCP(NCA), MT(ASCP), Florida Gulf Coast University, Fort Myers, FL 


\section{CLINICAL PRACTICE}

As we continue to see reductions in the number of clinical lab science programs around the United States, primarily due to the economic challenges that academic institutions are facing, Florida Gulf Coast University (FGCU) revised and reactivated its former $2+2$ NAACLS accredited program in 2006, by instituting an innovative $3+1$ approach, mainly through a strong partnership with the laboratory community. The cost reduction strategies involved; reducing 6 full-time faculty positions to 1 full-time faculty $/ 4$ adjunct faculty, and eliminating the costs of on-campus student laboratories entirely, by extending the length of internships (doubling) at the community laboratories. The program also changed traditional faceto-face didactic education to an entirely online format. The community laboratories also committed to fund the program for the first 3 years. Program expenses included: program director's salary, 5 adjunct salaries, mileage reimbursement for site visits to affiliates, and accrediting and licensing fees. Enrollment of 18 students covered these expenses. Two years after reactivating, the program enrolled 17 students and the following semester numbers increased to 19 . In the first year, 8 students graduated from the new program. Of the 8 students, 7 passed the ASCP certification exam and immediately became employed. In the face of our current economy, CLS programs are more at risk for termination these days. With the efforts of the community laboratories, their strong dedication and commitment, FGCU instituted an innovative structure to make its CLS program more viable by greatly reducing expenses of operation without sacrificing the quality of education.

Inclusion of Speaking and Writing Assignments into Upper-Level Medical Laboratory Science Courses

Mary F. Lux, PhD., MT(ASCP) and M. Jane Hudson, PhD., CLS(NCA), University of Southern Mississippi, Hattiesburg, MS

The University of Southern Mississippi recently instituted a Quality Enhancement Plan (QEP) as an accreditation requirement of the Southern Association of Colleges and Schools. The QEP required the inclusion of 2 graded speeches and generation of 5000 words in written assignments in two upper-level courses. The faculty developed a junior-level professional communication course that included both short writing assignments as critiques of posters and articles, as well as preparation of a lab procedure in the Clinical Laboratory Standards Institute format. Longer writing assignments included preparation of a fictional case study and an expanded research paper on student-chosen disease topics which included laboratory results that were used for diagnosis of disease. There were two graded PowerPoint oral presentations based on the case study and research paper. The faculty designed rubrics for assessment of speaking and writing assignments. Assessment by rubric in Professional Communication demonstrated an average improvement from $67 \%$ from the first writing assignment to $89 \%$ for the final writing assignment. Using pretests and post tests, $82 \%$ and $100 \%$ of Professional Communication students scored the same or higher on the post tests as compared with pretest scores, respectively. For the senior level courses, we developed a Capstone Experience with graded speaking and writing assignments in 4 practicum campusphase courses: Bacteriology, Hematology, Immunohematology, and Chemistry. These assignments comprise part of the grade for each course listed. By spreading the speaking and writing assignments through the senior practicum, the assignments have been successfully integrated into the curriculum.

Incorporating Corrective Measures to Instill Good Study Habits for Students Engaged in Distance Education

Gideon Labiner MS, MT(ASCP), CLS(NCA), Charity Einhaus Accurso PhD MT(ASCP), Melanie J. Giusti BS MT(ASCP), Lara N. Kolar BS MT(ASCP), Ryan D. McGough MS MT(ASCP), Erin C. Rumpke MS MT(ASCP), Linda J. Graeter PhD MT(ASCP), University of Cincinnati, Cincinnati, $\mathrm{OH}$

Students whose grade point averages fall below the benchmark of 2.5 enter the College of Allied Health Sciences' Academic Progression monitoring system. The CLS Faculty has developed a set of requirements to help this group of students succeed in the distance education learning environment. In the first phase, the program implemented a policy aimed at increasing examination scores. Students were required to complete a remediation activity should they fail to meet the benchmark of $75 \%$ on examinations. The remedial work entailed a review of the examination questions that each student missed. After implementing the policy, we noted that students' scores increased. In phase two in the summer and fall 2008 quarters, the program required this subset of students to attend the online office hours of the course director. The initial study showed a correlation between the number of chats attended and student improvement based on an increase in available points earned. Students who attended all of the chats scored $10 \%$ higher than those who attended half the chats or less. Since the pilot study, the program has worked with the college's Student Academic Advisors to implement this policy in all CLS Distance Learning courses. The efforts described above required the student to critically review material and to increase their faculty interactions in order to overcome previous comprehension deficiencies.

Job Satisfaction in Clinical Laboratory Sciences for Medical Technologists/Clinical Laboratory Scientists and Medical Laboratory Technicians/ Clinical Laboratory Technicians

Kathleen Finnegan MS, MT(ASCP)SH, State University of New York at Stony Brook, Stony Brook, NY

The purpose of this study is to evaluate the levels of job satisfaction among Clinical Laboratory Scientists and Clinical Laboratory Technicians and examine the factors influencing their satisfaction on a national level. In the winter of 2008 a twenty question survey was distributed electronically to 17, 587 Clinical Laboratory Scientists and Clinical Laboratory Technicians affiliated with the American Society for Clinical Pathology. The survey was received by 16, 306 (1,281 e-mails bounced) and 1,589 responses were obtained; which yield a response rate of $9.5 \%$. The participants that were chosen for this study have been in their field for $0-6$ years. The questions that were asked were: type of facility you are employed in, areas of the lab they worked in, which shift they worked, starting and current salary, satisfaction with pay, work schedule, work benefits, work environment, promotion possibilities, decision to enter profession and overall satisfaction. Results of the survey indicate that $25.7 \%$ are very satisfied with their profession, 55.3\% are satisfied, $15.6 \%$ are not satisfied and $3.5 \%$ are very unsatisfied. Factors such as current salary, promotion possibilities, opportunity for promotion, and 


\section{CLINICAL PRACTICE}

advancement showed lower levels of satisfaction. The data allowed for analysis of satisfaction and pay in the clinical laboratory sciences market and a comparison of satisfaction between CLS and CLT.

\section{Lab Buddies Mentor Program}

Linda Jeff, MA, MT(ASCP), Jessica Worthington, MS, MT(ASCP), University of Alabama at Birmingham, Birmingham, AL

First year CLS students usually must adjust to a large university setting as well as expectations of an intense, fast paced program. The Clinical Laboratory Sciences Student Association (CLSSA) initiated the Lab Buddies (LB) program to create one-on-one relationships to guide students so they could feel connected and gain information and skills needed for achieving success in the program. Second year students served as "Lab Buddy Leaders (LBLs)" for one or two students. Everyone completed information sheets, which were used to match LBs and LBLs. Duties of LBLs included serving as role models, motivating and supporting, solving problems, and helping LBs prepare for CLS curriculum. LBLs were required to have contact with buddy(ies) once a week, meet one-on-one every two weeks, provide nurturing and information, encourage participation in co-curricular activities including CLSSA, submit monthly reports, and refer LB to coordinator if necessary. Students were asked to evaluate the program after fall term. Eighty percent of respondents thought the LB program fulfilled or mostly fulfilled its purpose; $70 \%$ thought it should be continued, with the others undecided. Students thought most LBL duties/responsibilities were important, however, $30 \%$ thought monthly progress reports should be omitted and $50 \%$ thought meetings should be required less often. Comments included that the program should be less structured, students should be allowed to withdraw, and LBLs should not try to solve personal issues or share previous course materials. Overall, the LB program was viewed as successful; however, evaluations were used to revise the program for 2009-2010 students.

\section{Medical Insurance Requirements of CLS/MT College and University Programs \\ Amanda Evans, Jane Hudson, PhD, CLS (NCA), The University of Southern Mississippi, Hattiesburg, MS}

With the high cost of medical insurance and the fact that some students can not obtain medical insurance due to pre-existing health conditions and financial difficulty, this study addresses the current medical insurance requirements of clinical laboratory science programs located in the college or university setting. A survey, submitted to the Institutional Review Board (IRB) at the University of Southern Mississippi, was approved and sent to 121 NAACLS accredited CLS/ MT programs in the United States on September 15,2009 . The response rate was 68 percent. Data obtained from the surveys showed that 79 percent of CLS/MT programs require their students to have some type of medical insurance. The majority of the programs that require students to have medical insurance will not allow students to proceed into the clinical portions of their curriculum without it. In fact, 52 percent of programs require students to have medical insurance for both the classroom and clinical portions of their curriculum. Eighty-two percent of the programs offer a medical insurance plan for students. The cost of these plans generally range annually from $\$ 245$ to $\$ 2921$ per student. The information obtained from the survey can be used by CLS/MT programs directors to establish requirements and policies regarding medical insurance requirements for students.

\author{
Mixed Metaphor: Writing as a Learning Tool in Medical \\ Laboratory Science \\ Deborah D. Niven, MEd., MT (ASCP) and J. Michele Stuart, \\ PhD, MLS (ASCP),Associated Regional and University Pathologists \\ (ARUP) and University of Utah Salt Lake City, UT
}

This study used writing to help students learn content in a Medical Laboratory Science (MLS) class. Three different writing strategies were used in the research class. The effectiveness of the strategies was assessed with student created concept maps at the end of the course. Each student created a concept map illustrating the material learned during the course. The student maps were compared to a master map of course content. Maps were scored as a percentage according to specific criteria. Twenty-three of the twenty-eight student created maps contained more topics and more cross-links of topics for which there was a writing strategy used. Map analysis showed that $82 \%$ of the students showed more detail of knowledge for subjects which had a writing intervention and included more topics which had a writing experience on their maps. This finding supports the notion that students more thoroughly learned the topics for which they were more actively involved in the learning process through the writing assignments. The writing enabled a personal connection and engagement with the learning. The students expressed enthusiasm towards and favorable attitudes in learning about the course material in their writing assignments. This research can be used to foster student interest and success in the MLS field.

\section{A Novel Approach to Addressing Geriatrics in the Clinical Laboratory Science Curriculum \\ Cheryl Burns, MS, Linda A. Smith, PhD, Shirlyn B. McKenzie $\mathrm{PhD}$, Helen Sorenson MA, Martha Acosta, PhD, The University of Texas Health Science Center, San Antonio, TX}

The US Census Bureau projects that by 2030 there will be at least 69 million individuals 65 years of age or older. Few clinical laboratory science (CLS) programs have formally dealt with geriatric healthcare topics, yet graduates must be prepared to address the health care issues of this population. Shrinking resources, packed curricula, and a lack of faculty with expertise in geriatrics present problems with adding another new course. To address this problem, we invited several geriatric experts from other allied health disciplines to help design a symposium covering a number of agerelated health topics for CLS students. The resulting three hour symposium focused on the physiology, psychology, and demography of aging; specimen collection; changes in, and interpretation of geriatric laboratory test data; economic implications; and medical necessity. A video on communication skills was included. Student course evaluations averaged 4.6 on a 5-point Likert scale. The posttest scores on an aging quiz increased by $13 \%$ over pre-test scores. Students' comments were very positive. They found the overview of aging interesting and the communication techniques for working with the elderly and other healthcare professional helpful even for 


\section{CLINICAL PRACTICE}

their personal lives. In addition, students made a number of recommendations for other topics to be included. This symposium will be expanded for CLS and will form a template for development of an interdisciplinary geriatrics course in the School of Health Professions.

Patient safety-related concepts in CLS \& CLT curricula

Karen Golemboski, PhD, MT(ASCP), Bellarmine University, Louisville KY, and *Catherine Otto, PhD, CLDir(NCA), Oregon Health \& Science University Oregon Institute of Technology, Portland, OR

The ASCLS Patient Safety committee has been charged with the production of educational materials to incorporate patient safety into CLS and CLT curricula. A survey was developed to determine how concepts related to the six aims as described by the Institute of Medicine (safety, effectiveness, timeliness, efficiency, equitable care, and patient-centered care) are currently taught. The survey was sent to NAACLS-accredited CLS and CLT programs, both hospital- and university-based. Responses were received from 236 programs (56\% response rate). Results of the survey indicated that, although most programs $(70 \%)$ do not have a curriculum unit dedicated to patient safety, virtually all programs (97\%) teach some of the IOM's six concepts. For programs without a patient safety curriculum unit, the concept most commonly included in the curriculum was safety (doing no harm to the patient; 83\%), followed by timeliness and efficiency. The concept least likely to be included was effectiveness (49\%). Inclusion of concepts did not differ between CLS and CLT programs, but hospital-based CLS programs were more likely than university-based programs to include materials related to patientcentered care in the curriculum. A majority of the programs surveyed (80\%) indicated an interest in using new materials specifically developed to address the IOM areas of concern.

Personality Traits of Generation Y Medical Technologists: Implications for the Future

Janelle M. Chiasera, PhD, MT(ASCP), Rebecca A. Jones, MS, MT(ASCP), Linda Jeff, MA MT(ASCP), Pam Paustian, MS, University of Alabama at Birmingham, Birmingham, AL

The personality profile of medical technologists has remained unchanged over the past 30 years and suggests that professionals tend to be well suited for their current work paradigm. However, the Institute of Medicine has called for a radical redesign of the current heath system recommending movement away from a "silo" approach practicing medicine to a multiprofessional approach where the strengths and expertise of all health professionals are used to ensure high quality care. This will certainly require a change to the work paradigm of the future medical technologist. To date, no one has assessed the personality traits of students ready to enter the field to examine if their personality traits are suitable for this change. Medical Technology students in their senior year $(n=75)$ from 19 Universities across the country were asked to complete a survey to assess their personality traits using the Jung Typology questionnaire. An additional set of questions was developed to ascertain demographic information and to assess future career plans; however, the additional questions will not be linked to the personality type responses. The majority of subjects (64\%) were categorized as ENFJ (Teachers) and ENTJ (Fieldmarshals) which represents a departure from previous studies that categorized baby boomer medical technologists as primarily ISTJ (Protectors) and ISFJ (Inspectors). The change in personality type of generation $\mathrm{Y}$ medical technologists in our study suggests that this group of professionals is well suited and ready for the expanding and changing role of the future medical technologist.

Preparing Blood Bank Unknowns in a College Based Laboratory Setting Janet Oja, CLS (NCA), Weber State University, Ogden, UT

In a clinical laboratory sciences program where the laboratory simulation takes place in an academic setting, as opposed to a hospital based clinical rotation, preparing immunohematology unknowns is the student's first tangible introduction to performing routine Blood Bank procedures. The focus of this poster is to provide an overview of how that challenge is uniquely met at Weber State University. Local Blood Banks donate plasma with previously identified antibodies to the university which are prepared as unknown type and screens and antibody identification samples along with donated, outdated, packed red blood cells. Specific antibodies to which there is no donated plasma are created in the university laboratory with albumin, saline, anti-A, or anti-A,B, depending on the desired outcome, and then spiked with the appropriate antisera for the antibody being studied and identified. B plasma is purchased from the local Blood Bank due to the challenges associated with the blue color of commercially prepared anti-A antisera. Stimulating the students' critical thinking as they progress through the course is always the challenge when preparing unknown samples. Simulating laboratory unknowns with multiple antibodies, varying reaction phases, resolving discrepancies and ruling out antibodies are all concrete ways of accomplishing this task, along with traditional coursework. Preparing the students to perform these tasks in a practical setting as clinical laboratory scientists is the ultimate goal and always at the forefront of all unknown sample preparation.

Research and Scholarly Activity of Clinical Laboratory Scientists and Training Programs

JoAnn P. Fenn, M.S., MT(ASCP), University of Utah, Salt Lake City, UT; Vicki Freeman, PhD, MLS(ASCP), SC, FACB, University of Texas Medical Branch, Galveston, TX; Rebecca Laudicina, PhD, MLS(ASCP), The University of North Carolina at Chapel Hill, Chapel Hill, NC; Carol McCoy, PhD, MLS(ASCP), Fairview Health Services, Minneapolis, MN; Mary Ann McLane, PhD, MLS, University of Delaware, Newark, DE; Lillian Mundt, EdD, MLS(ASCP), Kaplan Higher Education, Chicago, IL; Joan Polancic, MSEd, MLS, ASCLS Director of Education and Project Planning, Bethesda, MD; Tim Randolph, PhD, MLS(ASCP), Saint Louis University, St. Louis, MO; Kristy Shanahan, MS, MT(ASCP), Rosalind Franklin University of Medicine and Science, North Chicago, IL

The ASCLS Research Taskforce emailed a survey to 7572 ASCLS members and CLS/CLT program directors to determine the state of 


\section{CLINICAL PRACTICE}

research in the profession, type of research conducted, funding, and research courses in CLS/CLT programs. The survey tool was Survey Monkey. Responses were received from 556 persons, with 55.5\% employed in clinical laboratory settings and $23.9 \%$ employed at two-year and four-year colleges and universities. Overall, 81.9\% (413) of respondents reported that research is not a required component of their employment. Barriers to conducting research were: time constraints (62.2\%); lack of funding (43.9\%); no opportunity (37.2\%;) lack of space or equipment (36.2\%). Respondents with Ph.D. degrees have been PIs or Co-PIs of research projects in higher frequency, receiving more than $\$ 15,500,000$ in research funding. The most frequent sources of funding from all respondents receiving research dollars were "Institutions" (75.2\%), "Private Foundations"(24.8\%), "NIH"(18.2\%), "Professional Organizations"(18.2\%), and "State" (13.2\%). Overall, the focus of research projects was reported as "Applied or Clinical"(49.3\%), "Education”(35.8\%), "Basic or Applied”(20.1\%), "Basic"(12.7\%). It is noteworthy that $57 \%$ of all respondents engage in scholarly activities, such as preparing and giving lectures and presentations. Also, CLS/CLT Program Directors reported that $77.7 \%$ do not have a separate research course for undergraduate students. Identifying research and scholarly activities of medical laboratory professionals and education programs provides insight for support of research activities and promotes the value of the profession.

\section{A Summer Externship Program: An Effective Recruitment Tool Carolyn Darr, MA, MT(ASCP), York Hospital, York, PA}

At York Hospital, the Clinical Laboratory Science Program and the Department of Laboratory Services wanted to encourage college students to major in clinical laboratory science by offering a summer externship program. The goal of the program was to develop an individual's interest in clinical laboratory science as a career option, thus ensuring that there was a group of students interested in the profession and in applying to the York Hospital Clinical Laboratory Science Program for their clinical year. With hospital approval, the Department of Laboratory Services offers summer employment to a limited number of college students. The criteria for selection includes college major, career goals, personal interview, and GPA. The externs are assigned and trained to work in one department. The departments that employ the externs are Blood Bank, Core Lab, and Microbiology. During the externship, the students become acquainted with a clinical laboratory in a hospital setting and become familiar with the varied roles of the clinical laboratory professionals. An aim of the program is to provide a link between the extern's classroom learning and Clinical Laboratory Science as a profession. Since its inception in 2001, the program has grown from 4 to 9 externs. From 2001 - 2006, 31 externs participated in the program; 13 were accepted into the CLS Program; and 7 are working at York Hospital. The success of this program can be determined by the number of externs that apply to the York Hospital CLS Program and that are employed by York Hospital after graduation.
Using Technology During a Natural Disaster to Keep the Learning Going

Jane Finley, MT(ASCP), University of Texas Medical Branch, Galveston, TX

Reports the coordination of a variety of technological tools used to continue the fall semester course work for first and second year students at University of Texas Medical Branch following Hurricane Ike. Incorporating each of these tools allowed students, faculty and staff to contact each other so plans to continue presenting lectures could be finalized. The use of social networking tools allowed students with basic needs to be connected with other students who could share supplies, shelter and books. It allowed the school's dilemma and needs to be communicated to the CLS community in the state and across the nation. It was used to capture and screen offers of help from clinical and academics sources. Using a course management system and an audio/video capture tool, online lectures were sent to all students until an alternate location for face to face lectures could be arranged. The accessibility and timely use of multiple technology functions found on the internet as well as mobile devices resulted in the return of all but one of the new students when classes resumed three weeks after the storm; all students were kept on track for intended learning units, the fall semester was extended only one week and the largest class to graduate in August 2009 "stopped for no storm".

\section{TECHNOLOGY DEMONSTRAIONS}

Teaching Laboratory Math and Statistics Online: Making the Most of Technology

Travis Price MS, MT(ASCP), Weber State University, Ogden, UT

Teaching laboratory mathematics and statistics in an online environment can be a challenging and time consuming experience. For any math or statistics course to be successful, students must be able to learn the concepts, obtain and submit assignments and receive feedback from an instructor in a timely manner. In the past this process for online students relied heavily on snail mail, fax machines or scanners and email. These methods for submitting assignments are extremely time intensive, prone to quality related issues and are often impeded by email attachment size limitations. Improvements in online delivery systems and digital media have changed the way laboratory math and statistics can be taught online. In the Applied Laboratory Math and Statistics at Weber State University as well as in the Advanced Laboratory Practices course students are able to view lectures, obtain homework assignments, submit their completed assignments, get feedback and receive pointers and tips for problems they might be struggling with, all without direct involvement from the instructor. To accomplish this, Flip Video ${ }^{\mathrm{TM}}$ cameras, Youtube $^{\mathrm{TM}}$ online video sharing as well as a few simple, but critical features of our university's online content delivery system, Blackboard, are used. This approach has not only reduced the amount of time and energy spent teaching these courses, but has also been hugely beneficial and timesaving for the students who take them. Student evaluations of the course before the implementation of the mentioned technology and afterward have been useful in assessing the way these changes have positively impacted students. 


\section{CLINICAL PRACTICE}

Using CellaVision in an Advanced Hematology Course

Kara Hansen-Suchy MEd, MT(ASCP)SH, Weber State University, Ogden, UT

Accurately assessing the morphology of blood cells is one of the most difficult procedures to perform in the clinical laboratory, as well as being one of the more frustrating and time consuming learning activities as a student. CellaVision ${ }^{\mathrm{n}}$ is a state of the art hematologic based competency software system initially designed to allow the laboratory the ability to evaluate the level of proficiency of its employees in the performance of manual differentials. The technology has been adapted to the educational setting in an advanced hematology course at Weber State University, as a tool to assess student performance on normal and abnormal manual differentials. The students participating in the use of CellaVision ${ }^{\text {st }}$ have all had a hematology class in which they learned how to do differentials using conventional microscope training. The unique feature that CellaVision "' provides enables the instructor to assess the competency of each student down to the individual cell level. This allows for easy identification of opportunities for discussion, additional education, as well as detection of students having difficulties in performing an accurate differential. The data analysis tool compares all participants against the expert. Students can view how their results compare to the expert allowing for self-education. This is a significant deviation from past methods of manual differential competency verification. It is like sitting down at a two headed scope with the entire class but better, because the results and graphs can be printed and stored, are comprehensive and can be easily utilized for numeric grades.

\author{
A Virtual Hemostasis Laboratory \\ Cheryl Swinehart, MS, CLS (NCA), University of Minnesota, \\ Minneapolis, MN
}

Traditionally, the University of Minnesota CLS hemostasis course has been taught using five wet labs: prothrombin time, partial thromboplastin time, thrombin time, factor assay and an inhibitor/inactivator study. Wet laboratory exercises are costly in that they require reagents, samples, and staff time for their preparation. Now that basic hemostasis testing has moved to instruments, few students will ever need to perform a hand clotting time. However, the theory and mechanics of the tests are important in order for students to troubleshoot and correctly interpret results. For these reasons, a virtual hemostasis laboratory was developed to take the place of the wet laboratory exercises in the hemostasis curriculum. Scripts were written for the online modules, which included course material and review questions. Videos were made of the clots forming and inserted within the modules. Students would click on a particular sample or control, watch the video, and click on the working virtual stopwatch on the screen when they saw the clot form. The students turned in their downloadable worksheet and took an online quiz with each virtual laboratory exercise. The students used the modules this spring and course evaluations showed they enjoyed the format. The students who performed the virtual tests had equal or slightly higher scores when given identical tests to students taught using wet laboratory exercises in previous years, although the difference was not statistically significant. Thus it seems the material was learned whether doing a wet lab or a virtual laboratory. 